\title{
Outstanding Outcomes from a Recent Theory of Gravity
}

\author{
Sandro Antonelli* \\ 151 A, S.P. 57 Accesso a M. 03017 Morolo Italy \\ *Corresponding author: antonelli41@live.it
}

Received November 20, 2014; Revised December 01, 2014; Accepted December 04, 2014

\begin{abstract}
This article intends to fathom a development by M. Tailherer which introduces a second gravitational equation devised to complete General Relativity (TGR), based on the ansatz of equating the curvature tensor opportunely contracted to the 4-vorticity by a new constant $S$ as measure of the intrinsic inertia of the curved SpaceTime. After justifying the need to deal with the 2nd fundamental tensor in Relativity in discussing the dynamics of Space-Time structure, it has been shown how the model exhibits unforeseen analogies with the electromagnetic theory. As direct continuation of the analysis of the gravitational wave propagation in free space, it has been seen that on asymptotic conditions the polarization state can be retrieved as mixture of two independent modes likewise TGR as from harmonic constraints on the homogeneous solution of wave equation. Actually, in this gravitational framework, at least for one polarization state, transverse waves propagate causing equal in-phase deformation displacement, not counter-phase as expected in gravitational interferometry experiments at present status. Computation of gravitational power losses for the keplerian system B 1913+16 in the solution by approximations of the inhomogeneous problem has been carried out to the 1st order, which has allowed the assessment of a new universal gravitational constant for the first time ever.
\end{abstract}

Keywords: General Relativity, $2^{\text {nd }}$ fundamental tensor, gravitational waves, Tensorial Curl, 4-vorticity

Cite This Article: Sandro Antonelli, “Outstanding Outcomes from a Recent Theory of Gravity." International Journal of Physics, vol. 2, no. 6 (2014): 267-276. doi: 10.12691/ijp-2-6-10.

\section{Introduction}

Despite its impressive success [10], linear approximation in TGR seems to contradict in that the exact theory would not on principle entail emission of gravitational radiation i.e. waves physically meaningful as pointed out by some authors [14]1. Other alternatives have then been explored to search for the mechanism responsible of gravitational wave genesis. Since General Relativity describes the universe in terms of an hypersurface metric of space-time (the V4 chronotope), and since a surface is characterized differentially by the two Gauss' quadratic forms (the components whereof of the $1^{\text {st }}$ one being the coefficients of the metric), it follows that the single Einstein's equation is insufficient to determine the evolution of the cosmos. Indeed two tensorial equations are needed in two unknown tensorial variables. Certainly, as far back as pioneering studies on the evolution of surfaces, one might wonder it has taken so long to understand such a simple thing, but physics up to now could not imagine the 4-vorticity $\omega_{\mu v}$ related to the tensor of curvature, pivot of Tailherer's theory, because the quantity $S$ that links them, that we shall rate, lacked among the fundamental constants. Actually, the analysis of the motion of a continuum has shown a resemblance with Space-Time that might be more than superficial, for

1 The simplest argument is that any moving body will travel a geodesic line and that will do without gravitational losses. it has been possible to establish a valuable equation by making use of a 4-dimensional extension of the vortex gradient encountered in lagrangian description of continua $[3,4]$ relating the angular velocity tensor to the deformation tensor $K_{\mu v}$. In particular, the ansatz has been tried thereby the presence of 4-vorticity does also mean the presence of curvature of space time through the constant $S$, which has shown itself fundamental of all reasoning bringing to the gravitational wave propagation scenario in this model. I want to stress that this approach is not a reformulation of TGR in its hamiltonian formalism as in the Arnowitt-Deser-Misner decomposition nor in its content of dynamic evolution of a time-like congruence as in Raychaudhuri [16] identity but relies on an additional equation involving vorticity and a new physical constant, not a PPN-like factor or a Lagrange multiplier.

\subsection{The $2^{\text {nd }}$ Fundamental Tensor $K_{i j}$ and the a New Gravitational Equation}

First of all, we show how the coefficients $2 \mathrm{Kij}$ of the $2^{\text {nd }}$ differential form of a surface $\sigma$ are directly related to the derivative of metric with respect to a parameter (the proper time in the case of relativity). For this purpose, once fixed a coordinate reference system upon the surface, the $K i j$ 's are related to the basis vectors on the surface relative to the point $P: \boldsymbol{e}_{i}=\partial \boldsymbol{O P} / \partial x^{i}(i=1,2)$ - whence the

2 In the Gauss' original notation these are denoted by the letters $\mathrm{L}=\mathrm{K}_{11} ; \mathrm{M}=\mathrm{K}_{12} ; \mathrm{N}=\mathrm{K}_{22}$. 
metric tensor $g_{i j}=\boldsymbol{e}_{i} \cdot \boldsymbol{e}_{j}$ - through the differential link : $\partial_{i} \boldsymbol{e}_{j}=\Gamma_{i j}^{m}+\boldsymbol{e}_{m_{0}} K_{i j} \boldsymbol{v}$ with $\boldsymbol{v}=\left(\boldsymbol{e}_{1} \times \boldsymbol{e}_{2}\right) /\left\|\boldsymbol{e}_{1} \times \boldsymbol{e}_{2}\right\|$ normal versor to the surface and so to the basis vectors, and $\Gamma_{i j}^{m}$ the Christoffel symbols of $2^{\text {nd }}$ kind. This definition assures consistency with well-known consequences, above all the symmetry of $K_{i j}$ by the Schwartz theorem $\partial_{i} \boldsymbol{e}_{j}=\partial_{j} \boldsymbol{e}_{i}$ and the equivalence of the first two invariants of $K_{i n} g^{n j}$ with the mean and Gauss' curvature as plainly inferred in [1]. Multiplying by the direct product the former will yield:

$K i j=\partial_{i} \boldsymbol{e}_{j} . \boldsymbol{v}=\partial_{i}\left(\boldsymbol{e}_{j .} . \boldsymbol{v}\right)-\boldsymbol{e}_{j .} . \partial_{i} \boldsymbol{v}=-\boldsymbol{e}_{j .} . \partial_{i} \boldsymbol{v}$. Now, taking the generic surface $\sigma^{\prime}$ parallel to $\sigma$ hence defined by $\boldsymbol{O} \boldsymbol{P}^{\prime}=$ $\boldsymbol{O P}+\tau \boldsymbol{v}$, and on applying the definition $\boldsymbol{e}_{i}^{\prime}=\partial_{i} \boldsymbol{O P} \boldsymbol{P}^{\prime}=\boldsymbol{e}_{i}+$ $\tau \partial_{i} \boldsymbol{v}$ we have for the metric of the parallel surface $g_{i j}^{\prime}=$ $\boldsymbol{e}_{i .}^{\prime} . \boldsymbol{e}_{j}^{\prime}=g_{i j}-2 \tau K i j+\tau^{2} \operatorname{Kim} K^{m} j$ and to the limit as $\tau->0$, i.e. at less of $\tau^{2}$ order terms: $K i j=-1 / 2 \partial_{\tau} g_{i j}$. Thus, the second quadratic form $K i j d x^{i} d x^{j}$ tells us how the metric varies on passing to a parallel surface infinitely nearby, the sign in front being unessential on account of the arbitrariness of the choice of the normal versor (as implied by the local pseudo-invariance of the $2^{\text {nd }}$ differential form). As elicited further, Kij can be related to the deformation speed tensor in continuum mechanics. By his ansatz [8] Tailherer has been able to get a second gravitational equation ( $\nabla_{\mu}$ is the covariant derivative) linking the nabla of skew-symmetric curvature tensor $C_{\mu v}$ (hereafter the Riemann $C$ Tensor) with the curl of the deformation tensor of the metric:

$$
\nabla_{\sigma} \omega_{\mu v}=\nabla_{\mu} K_{v \sigma}-\nabla_{v} K_{\mu \sigma}(\mu, v, \sigma=0,1,2,3) 3
$$

$\mathrm{C}_{\mu v}=\mathrm{S} \omega_{\mu \nu}$ with $\mathrm{C}_{\mu \nu}=\mathrm{R}_{\alpha \beta \mu \nu} \epsilon^{\alpha \beta}$ from which

$$
\begin{aligned}
& { }^{(1)} C_{\mu \nu}={ }^{(1)} R_{\alpha \beta \mu v} \in{ }^{\alpha \beta} \\
& =-k\left(g_{\alpha v} / 4\right)\left(T_{\beta v}-1 / 2 g_{\beta \mu} T\right) \epsilon^{\alpha \beta}
\end{aligned}
$$

with ${ }^{(1)} R_{\alpha \beta \mu v}=g_{\alpha v} R_{\beta \mu} / 4$ curvature tensor in its first approximation estimate owing to $R_{\beta \mu}=g^{\alpha v} R_{\alpha \beta \mu \nu}$, as starting position in coming by $C_{\mu \nu} ; \epsilon^{\alpha \beta}=1$ for $\alpha, \beta$ consecutive indexes and $\epsilon^{\alpha \beta}=-\epsilon^{\beta \alpha}$ (see Eq.(25) further); $k=8 \pi G / c^{4}$. We note that if the energy momentum tensor in matter $T_{\mu v}$, expressed through the Riemann tensor by Einstein's equations in accordance with Eq. (2), does not depend on the metric coordinates - isotropic case -, we have a null LHS in (1), thus retrieving the MainardiCodazzi condition [1] for the coefficients of the $2^{\text {nd }}$ quadratic form expressing its propriety of symmetry with respect to the covariant derivative. And just because of the covariant derivative on the LHS, a cosmological term is immaterial at this approximation of the theory. As said by Tailherer, Eq.(1) would play the role of Faraday's law in Relativity. By a double curl the equation of waves comes out. Actually, by performing another curl (rotor) of Eq. (1) we get ( [8] Eq. (4.18)):

$$
\begin{aligned}
& K_{\mu v / \alpha}^{\alpha}-K_{\mu v / \mu}^{\alpha} \\
& =\left(|\nabla|^{2} K\right)_{\mu v}-(\operatorname{grad} \operatorname{div} K)_{\mu v} \\
& =-K_{\mu}^{\alpha} R_{\alpha v}+K_{\beta}^{\alpha} R_{v \alpha \tau \mu} g^{\beta \tau}-[1 /(2 S)] C_{\mu \alpha v^{\prime}}^{\alpha}
\end{aligned}
$$

3 It apparently satisfies the Bianchi identity $\nabla_{\sigma} \omega_{\mu \nu}+\nabla_{\mu} \omega_{v \sigma}+\nabla_{\nu} \omega_{\sigma \mu}=0$ and because of skew-symmetry of $\omega_{\mu \nu}$ covariant derivatives may be substituted for ordinary ones. with $R_{\alpha \sigma}$ the symmetric Riemann tensor and the slash standing for covariant or contravariant derivative whether put down or up.

\section{Methods}

\subsection{Physical meaning of the main equation}

The previous Eq. (1) has respectively a cinematic, dynamic and geometric content, in particular the last one states that a variation of curvature manifest in a propagation of metric deformation or, as it will be presently seen, in a "gravitational current" in the Space-Time. For this purpose let us call total dynamic flux crossing a two dimensional oriented surface $\Sigma$ of parametric equations $x^{\mu}(r, s)$ and complete contour $l$, the double covariant integral with respect to the skew indexes $\mu, v$ (making use of the equality $\nabla_{\mu} K_{v \sigma}-\nabla_{\nu} K_{\mu \sigma}=\partial_{\mu} K_{v \sigma}-\partial_{\nu} K_{\mu \sigma}$ (see Eq. 4.8 of [8])) :

$$
\begin{aligned}
& \Phi_{\Sigma}(\text { curl K })=\iint \sum_{\mu<v}^{0,3}\left(\nabla_{\mu} K_{v \sigma}-\nabla_{v} K_{\mu \sigma}\right) \\
& \times\left(\frac{\partial x^{\mu}}{\partial r} \frac{\partial x^{v}}{\partial s}-\frac{\partial x^{v}}{\partial r} \frac{\partial x^{\mu}}{\partial s}\right) d r d s \\
& =\frac{1}{2} \iint\left(\partial_{\mu} K_{v \sigma}-\partial_{v} K_{\mu \sigma}\right)\left(\frac{\partial x^{\mu}}{\partial r} \frac{\partial x^{v}}{\partial s}-\frac{\partial x^{v}}{\partial r} \frac{\partial x^{\mu}}{\partial s}\right) d r d s
\end{aligned}
$$

Then the Green-Stokes theorem states that the previous expression equals the dynamic circulation of $K_{\mu \sigma}$ along the circuit $l$ namely the integral:

$$
J_{\sigma}=\oint_{l} K_{\mu \sigma} d x^{\mu}
$$

We have therefore in our case that the "flux" of the derivative of the $C$ Riemann tensor is:

$$
\Phi_{\Sigma}\left(\nabla_{\sigma} C_{\mu v}\right)=S J_{\sigma}
$$

If we let the surface be far enough from the masses distribution so that it allows us to consider the Space-Time nearly flat, we may take the derivative out of the sign of the integral to get:

$$
\nabla_{\sigma} \Phi_{\Sigma}\left(C_{\mu v}\right) \approx S J_{\sigma}
$$

Surprisingly we recognize in the preceding expression the striking formal resemblance with the Faraday Neumann law of electromagnetism in which the magnetic field and the current take respectively the place of the $C$ Riemann tensor and the "gravitational current" $J_{\sigma}$. Thus we can state that a variation of "flux" of the energy momentum tensor through Eq. (2) or (24) at first approximation of the theory, must manifest in an induced gravitational current of gravitational radiation. After introducing the strength tensor $\Phi_{v \sigma \mu}=K_{v \sigma / \mu}-K_{\mu \sigma / v}$, from which the free Lagrangian $L=1 / 4 \Phi_{\alpha \sigma \rho} \Phi^{\alpha \sigma \rho}$, the analogy with electromagnetism looks clear, yet with $K_{\mu v}$ playing the role of a $2^{\text {nd }}$ rank potential referring to 2-spin graviton. In the following sections we shall deal with the vortex 4-dimensional formula going deeply into the resolution of a well-studied gravitational binary system. 


\subsection{The Deformation Tensor of the Metric and the 4-Vorticity}

As in the case the surfaces, let us consider now all the points of Space-Time parametrized with their co-ordinates which define the position vector $\boldsymbol{O P}$ in a 4-dimensional differentiable variety and a local frame relating to a local basis of vectors at each point of the chronotope $\boldsymbol{e}_{\alpha}=$ $\partial \boldsymbol{O P} / \partial x^{\alpha}$ wherefrom the metric tensor $g_{\alpha \beta}=\boldsymbol{e}_{\alpha} \cdot \boldsymbol{e}_{\beta}$. While in Special Relativity $g_{\alpha \beta}$ is a constant tensor, in TGR we think of $g_{\alpha \beta}\left(x^{\mu} / \tau\right)$ as function of the variables $x^{\mu}$ and $\tau$ so as $\boldsymbol{e}_{\alpha}$ too. For simplicity's sake, let us restrict ourselves to a sub-space of $g_{\alpha \beta}$ spanned by any tern of vectors $\boldsymbol{e}_{\alpha}$. It follows that if the relations hold in each subspace, as they do, they hold in all the 4-dimensional space for any inferred equation presenting three indexes or less. Therefore, let us choose without loss of generality the tern referring to the space indexes $h=1,2,3$. Consider now the gradient of the space components of 4-velocity which will be of the type (the $c$ factor is adopted ease of dimension):

$$
\partial_{h} v=c q_{h k} e^{k}\left(\partial_{h}=\partial / \partial x^{h} \quad h, k=1,2,3\right)
$$

The matrix $q_{h k}$ can always be split up in two parts

$$
q_{h k}=\left(\partial_{h} v \cdot e_{k}\right) / c=K_{h k}+\omega_{h k}
$$

with symmetrical part the deformation velocity of the metric

$$
\begin{aligned}
& K_{h k}=1 / 2 c\left(\partial_{\tau} e_{h} \cdot e_{k}+\partial_{\tau} e_{k} \cdot e_{h}\right) \\
& =1 / 2 c \partial_{\tau}\left(e_{h} \cdot e_{k}\right)=1 / 2 c \partial_{\tau} g_{h k}
\end{aligned}
$$

which can be referred to the 2nd fundamental tensor as already outlined, and the skew-symmetric part

$$
\omega_{h k}=\left(\partial_{h} v \cdot e_{k}-\partial_{k} v \cdot e_{h}\right) / 2 c=-\omega_{k h} .
$$

Following the classical approach presented in the Ferrarese's works [4], we thus get Eq.(2) restricted to the three-dimensional space and therefore readily generalized to the whole Space-Time according to our reasoning. Let us then differentiate $\boldsymbol{e}_{\mu}\left(x^{v} / \tau\right)$ with respect to $x^{v}$, then we get for definition of Christoffel symbols [4]: $\partial_{\nu} \boldsymbol{e}_{\mu}=\Gamma^{\alpha}{ }_{v \mu} \boldsymbol{e}_{\alpha}$. From that it turns out that $\partial_{\nu} v=\partial_{\nu}\left(v_{\mu} \boldsymbol{e}^{\mu}\right)=\left(\partial_{v} v_{\mu}-\Gamma^{\alpha}{ }_{v \mu}\right.$ $\left.v_{\alpha}\right) \boldsymbol{e}^{\mu}=\left(\nabla_{v} v_{\mu}\right) \boldsymbol{e}^{\mu}$ leading Eq.(11) to the expression:

$$
\begin{aligned}
\omega_{\mu v}= & \left(\nabla_{\mu} v_{v}-\nabla_{v} v_{\mu}\right) / 2 c \\
& =\left(\partial_{\mu} v_{v}-\partial_{v} v_{\mu}\right) / 2 c
\end{aligned}
$$

taking advantage of the symmetry of Christoffel symbols with respect to inferior indexes.

We may put the previous expression in a form evidencing the curl of the 4-velocity:

$$
\Omega^{\alpha \beta}=\varepsilon^{\alpha \beta \mu v} v_{v / \mu}
$$

with $\varepsilon^{\alpha \beta \mu v}$ Levi-Civita tensor. Thus (12) is nothing but the relativistic version of the well-known relation of hydrodynamics $\boldsymbol{\omega}=1 / 2$ curl $\boldsymbol{v}_{E U L}$ with $\boldsymbol{v}_{E U L}$ eulerian velocity to which the known generalized Lagrange and Helmoltz theorems can be applied [2,5]. In particular, standing the identity of Eq. (2) advanced at the beginning between the vortex and the skew Riemann tensor, the
Lagrange theorem 4 maintains that if present, a gravitational field can never destroy itself and if absent can never originate.

\subsection{Characteristics of Deformation}

The analysis of the deformations of metric is very interesting in that we may infer remarkable proprieties of the solution of the differential system (2) without passing through its resolution but by only qualitative analysis. Indeed, by taking a free falling frame and assuming in a fixed point $P^{*}$ initial geodesic coordinates we find the finite deformation of the metric at the $\tau$ proper time to be 5 :

$$
\begin{aligned}
& 2 c \int_{\tau}^{\tau} * K_{\mu \nu}\left(x^{\rho} / \tau^{\prime}\right) d \tau^{\prime} \\
& =\mathrm{g}_{\mu v}\left(x^{\rho} / \tau\right)-\eta_{\mu v}=\mathrm{h}_{\mu \nu}\left(x^{\rho} / \tau\right)
\end{aligned}
$$

with $\eta_{\mu \nu}$ Minkowski tensor. Because of the Gauss gauge $g_{00}=1, g_{0 k}=0(k=1,2,3)$, it follows at once from Eq.(14) that $h_{00}=h_{0 k}=0$, and hence $K_{00}=0, K_{0 k}=0$. Moreover, we note $h_{\mu v}$ to depend only on the initial condition and on the displacement in terms of chronotope co-ordinates $\boldsymbol{s}=$ $\boldsymbol{x}-\boldsymbol{x}_{0}$. In fact, let us try to put Eq. (14) in function of the displacement; since $\boldsymbol{O P}=\boldsymbol{O} \boldsymbol{P}^{*}+\boldsymbol{s}$ let us see what happens to the local base at the point $P$ :

$$
\mathrm{e}_{\mu}=\frac{\partial \mathrm{OP}}{\partial x^{\mu}}=\mathrm{c}_{\mu}+\frac{\partial \mathrm{s}}{\partial x^{\mu}}
$$

with $\boldsymbol{c}_{\mu}$ rectangular base vectors such that $\boldsymbol{c}_{\mu} . \boldsymbol{c}^{v}=\delta_{\mu}{ }^{v}$.

Then, remembering that $g_{\alpha \beta}=\boldsymbol{e}_{\alpha \cdot} \cdot \boldsymbol{e}_{\beta}$, Eq.(14) becomes:

$$
h_{\mu v}\left(x^{\rho} / \tau\right)=\left(\frac{\partial \mathrm{s}}{\partial x^{\mu}} \cdot \mathrm{c}_{v}+\frac{\partial \mathrm{s}}{\partial x^{v}} \cdot \mathrm{c}_{\mu}\right)+\frac{\partial \mathrm{s}}{\partial x^{\mu}} \cdot \frac{\partial \mathrm{s}}{\partial x^{v}}(16 \mathrm{a})
$$

Since $\boldsymbol{s}=s_{\alpha} \boldsymbol{c}^{\alpha}=s^{\alpha} \boldsymbol{c}_{\alpha}$ Eq. (16a) reads:

$$
h_{\mu v}\left(x^{\rho} / \tau\right)=\left(\partial_{\mu} s_{v}+\partial_{v} s_{\mu}\right)+\partial_{\mu} s_{\alpha} \partial_{v} s^{\alpha} \text {. }
$$

Thus, the characteristics of deformation consist of two terms whose the first, linear and symmetric in the derivatives of the displacement, occurs in infinitesimal problems well-studied in deformations of Elasticity [6,7] and that the reader is also addressed to for the analysis of the effects of the gravitational wave on the matter.

That two polarization modes can be retrieved as in TGR, it can be seen by considering the solution of the associated homogeneous one of Eq.(3) as discussed in $\$ 3.1$ in the asymptotic condition of weak field so that an euclidean metric may be taken. Of course this would not be strictly exact in that $g_{\mu \sigma}=$ const would imply $K_{\mu \sigma}=0$ but this makes no pretence of rigour. Substituting $K_{\mu \sigma}=\varphi_{\mu \sigma}+$ $x_{0} \psi_{\sigma / \mu}$ into Eq.(3), with $\varphi_{\mu \sigma / \alpha}{ }^{\alpha}=\psi_{\sigma / \alpha}{ }^{\alpha}=0$ (harmonic functions), it is found (see [8] Eq. 4.22) that6 [ $\psi_{\sigma / 0}-$ $\left.\varphi_{\alpha \sigma}{ }^{/ \alpha}\right]_{/ \mu}=0$, i.e. the term in brackets is a constant 4vector that has been chosen to be null to guarantee the

4 Although proved by reduction to absurdity from the flat space-time case, a demonstration in general coordinates has not given yet.

5 We should not be surprised at noting the fundamental tensor $g_{\mu v}$ to be inferred from a second quadratic form $K_{\mu v}$ not more than in kinematics the position elicited from the velocity through integration of the law of motion.

6 This is no longer true in general coordinates because the Schwartz theorem of crossed derivatives does not hold. 
right behaviour to infinity, which amounts anyway to 4 conditions, that summed up to the as many ones of Gauss's gauge reduce the 10 components of $K_{\mu v}$,viz. the number of physical degrees, to just two different polarizations.

For a wave propagating along the $\mathrm{z}$ axis, that is to say, perpendicularly to the direction of observation of a free falling frame (so that expecting $h_{z z}\left(x^{\rho} / \tau\right)=0$ if we consider $s_{3} \equiv 0$ in Eq.(16b) to the first order ), we may take the two linear independent wave polarizations, say

$$
A_{\oplus}=\left[\begin{array}{llll}
0 & 0 & 0 & 0 \\
0 & 1 & 0 & 0 \\
0 & 0 & 1 & 0 \\
0 & 0 & 0 & 0
\end{array}\right]
$$

for deformations along the axes and

$$
A_{\otimes}=\left[\begin{array}{llll}
0 & 0 & 0 & 0 \\
0 & 0 & 1 & 0 \\
0 & 1 & 0 & 0 \\
0 & 0 & 0 & 0
\end{array}\right]
$$

for angular deformations of axes directions 7.

It may be shown [6,7] that after the deformation the angle between the axes $r$ and $s$ is given by:

$$
\Theta_{r s}=\frac{\pi}{2}-\arcsin \frac{h_{r s}}{\sqrt{1-h_{r r}}+\sqrt{1-h_{s s}}}
$$

so the presence of off diagonal elements in the matrix $h$ does mean angular deformations between axes directions.

Moreover, because the lengthening of any vector $\boldsymbol{O A}_{0}$ (the versor of which be $\boldsymbol{a}_{0}$ ), into $O A$ is determined by the linear dilatation coefficient [6] :

$$
\delta_{a}=\frac{\overline{O A}-\overline{O A_{0}}}{\overline{O A_{0}}}=\sqrt{1-h_{h k} a_{0}^{h} a_{0}^{k}}-1
$$

we may note that the polarization $A_{\otimes}$ breaks any cylindrical symmetry since would involve opposite length variations in any perpendicular rotated directions having $\boldsymbol{a}_{0}$ as axis e.g.

$$
a_{01}:\left[a_{0 x}, a_{0 y}\right] \text { and } a_{02}:\left[-a_{0 y}, a_{0 x}\right] \text {. }
$$

\section{Results}

\subsection{Homogeneous Solution}

In order to work out the "gravitational billow", we now refer to the homogeneous solution 8 as the proposed one of (4.21) of [8] $K_{\mu \nu}\left(x^{\rho}, x^{0}\right)=\varphi_{\mu \nu}+x^{0} \psi_{v / \mu}$ in the approximation of quasi- cartesian coordinates, that is to say, substituting the covariant derivatives by the ordinary ones and trying to find the solution with regard to symmetric cylindrical boundary conditions. Hence, let us consider a wave of $A_{\oplus}$ type. Thus, using the harmonic function

7 Other combinations of sign as the polarization $A_{11}=-A_{22}=1$ lead to volume conservation according to Eq. (17) which evidently is not what we expect.

8 To simplify the working out the Fourier expressions we do a Wick rotation $x^{0}=$ ic $\tau$ thus making euclidean the Minkowski tensor and imaginary the initial deformation velocity $\varphi_{\alpha \beta}=1 / 2 \partial g_{\alpha \beta} /\left.\partial(i c t)\right|_{0 .}$. At the end of calculation one has to take the imaginary part changed of sign.

$$
\varphi_{\mu v}=\mathrm{A}_{\oplus} \frac{\Pi(\rho-c t)}{\rho}
$$

with $\Pi$ any function, we get the following initial condition:

$$
K_{\mu v}(t=0)=\varphi_{\mu v}(t=0)=\mathrm{A}_{\oplus} f(\rho)=f_{\mu v}(\rho)
$$

Then, having expressed the harmonic function by Fourier integral

$$
\varphi_{\mu \nu}(\mathrm{x}, \tau)=\int F_{\mu \nu} e^{i c \tau \delta+i\left(\alpha x_{1}+\beta x_{2}+\gamma x_{3}\right)} d \alpha d \beta d \gamma(20 \mathrm{a})
$$

and having introduced spherical co-ordinates, such that $\alpha x^{1}+$ $\beta x^{2}+\gamma x^{3}=\rho r \cos \theta$, that is to say, taking the distance vector along the direction of propagation $z=x^{3}$ and $d \alpha d \beta d \gamma=\rho^{2} \sin \theta d \rho d \theta d \varphi$ the following transforms are calculated :

$$
\begin{aligned}
F_{\mu \nu}(\alpha, \beta, \gamma) & =\frac{1}{(2 \pi)^{3}} \int f_{\mu \nu} e^{i(\alpha \varsigma+\beta \eta+\gamma \chi)} d \varsigma d \eta d \chi \\
& =\mathrm{A}_{\oplus} F
\end{aligned}
$$

and:

$$
\begin{aligned}
K_{x x}=K_{y y} & =\int\left\{F(\alpha, \beta, \gamma)+i\left(\frac{i c \tau \alpha}{\delta}\right)[i \alpha F(\alpha, \beta, \gamma)]\right\} \\
& \times e^{i c \tau \delta+i\left(\alpha x_{1}+\beta x_{2}+\gamma x_{3}\right)} d \alpha d \beta d \gamma
\end{aligned}
$$

( $\delta=-\left(\alpha^{2}+\beta^{2}+\gamma^{2}\right)^{1 / 2}$ because of the harmonic condition, namely the usual relation between frequency and wave number components $\omega=c \delta$ ).

Then, having considered the imaginary part, sign changed, after the integration, the relative deformations along the two axes will be:

$$
\begin{gathered}
h_{x x}(r)=h_{y y}(r)= \\
2 c \int_{0}^{\tau} K_{11}\left(x^{\rho} / \tau^{\prime}\right) d \tau^{\prime}=2 c \int_{0}^{\tau} K_{22}\left(x^{\rho} / \tau^{\prime}\right) d \tau^{\prime}
\end{gathered}
$$

with $r$ the distance between source and observer. Even examples with simple initial conditions entail cumbersome expressions involving delicate problems of convergence. So we skim over.

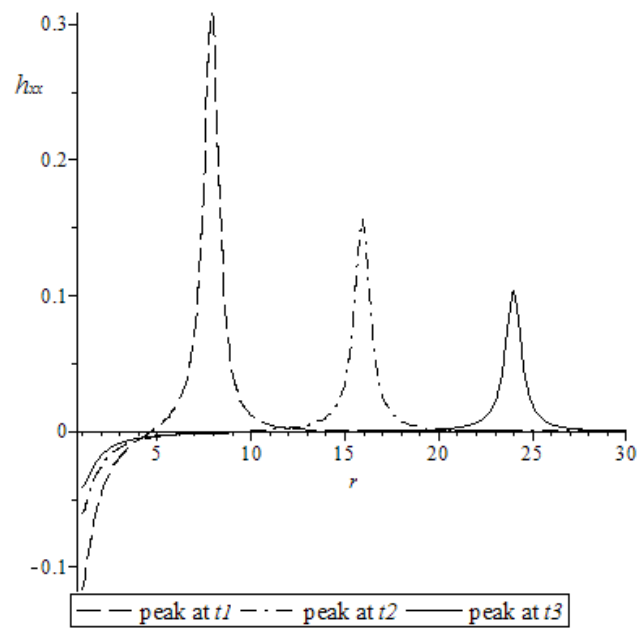

Figure 1. Plot in function of $r$ (arbitrary units) of the characteristic gravitational billow wave shape solution (numerical) $h_{x x}$ with respect to a discontinuity source located about $r=0$ (e.g. $\left.K_{x x}(t=0)=1 /\left(1+r^{2}\right)^{3 / 2}\right)$ at fixed times $t 1<t 2<t 3$ with its attenuating moving peak at $r=c t$ 


\subsection{Non-homogeneous Solution in Gravitational Energy Losses of a Punctual Keplerian System}

By referring back to [8] we see the principal problem to be related to the following integral:

$$
K_{\mu \sigma}=\int N_{\sigma \alpha \mu}^{\beta} y_{\beta}^{\alpha} d^{4} \xi+y_{\mu \sigma}
$$

where the integral runs over the coordinates $\xi$ of the an astrophysical source where the energy-momentum tensor is not null. Hence $d^{4} \boldsymbol{\xi}=d^{3} \boldsymbol{x}^{\prime} d \tau$ with $\boldsymbol{x}^{\prime}$ space coordinates of the keplerian system under discussion (binaries self-gravitating stars,...). The kernel and the other term result to be:

$$
N_{\sigma \alpha \mu}{ }^{\beta}=3 /\left(2 \pi^{2}\right)\left[\begin{array}{l}
\left(\delta_{\mu}{ }^{\beta} R_{\alpha \sigma}+R_{\sigma \alpha}{ }^{\beta}{ }_{\mu}\right) / r^{2} \\
+\nabla_{\mu}\left(1 / r^{2}\right)\left(\xi^{\rho}-x^{\rho}\right) \\
\times\left(\delta_{\rho}{ }^{\beta} R_{\alpha \sigma}-R_{\sigma \alpha}{ }^{\beta} \rho\right)
\end{array}\right]
$$

with $R_{\alpha \sigma}$ symmetrical Riemann tensor $9, r$ is the chronotope distance from the observation point $\boldsymbol{x}$ to the source point $\xi$ and

$$
\begin{aligned}
& y_{\mu \nu}=-3 \frac{1}{4 S r^{2} \pi^{2}} \int\left[C_{\mu \nu} \sigma^{v}\right. \\
& \left.+\nabla_{\mu}\left(\frac{1}{r^{2}}\right) \cdot\left(x^{\rho}-\xi^{\rho}\right) C_{\rho v}{ }^{\prime} \sigma^{v}\right] d^{4} \xi .
\end{aligned}
$$

Moreover, as said before, by the attempting of estimate of $R_{\mu v \rho}$ as ${ }^{(1)} R_{\mu v \rho \sigma}=g_{\mu \sigma} R_{v \rho} / 4$ as starting position in getting ${ }^{{ }^{11} C_{\mu v}}$ in a successive approximations process, we have according Eq.(2) for the "skew" energy momentum tensor:

$$
Y_{\mu v}=-\left(g_{\alpha v} / 4\right)\left(T_{\beta \mu}-1 / 2 g_{\beta \mu} T\right) \epsilon^{\alpha \beta}
$$

with $T_{\beta \mu}$ einstenian energy-momentum tensor of a punctual keplerian system.

As in [8], we pick out the generic skew-symmetric cartesian tensor $\epsilon^{\alpha \beta}$.

$$
\epsilon^{\alpha \beta}=\left[\begin{array}{cccc}
0 & 1 & 0 & 0 \\
-1 & 0 & 1 & 0 \\
0 & -1 & 0 & 1 \\
0 & 0 & -1 & 0
\end{array}\right] \text { and } \in_{\alpha \beta}=\left[\begin{array}{cccc}
0 & -1 & 0 & -1 \\
1 & 0 & 1 & 0 \\
0 & -1 & 0 & -1 \\
0 & 0 & -1 & 0
\end{array}\right]
$$

as the inverse matrix.

It is apparent in the previous ones Eq.(23)s that we may neglect the derivative of the inverse squared of $r$ on account of the very large astronomical distance between source and observer and so bring $r$ out of the integral sign. Besides, we may put in the last expression $C_{\mu v / \sigma}{ }^{v}$ in the following form by means of the Bianchi identity (footnote 3) thus evidencing a curl: $C_{\mu v / \sigma}{ }^{v}=-C_{\nu \sigma / \mu}{ }^{v}+C_{\mu \sigma / v^{v}}=-1 / 2$ $\varepsilon_{\mu \alpha \delta \gamma} \varepsilon^{\delta \gamma \kappa \lambda} C_{\lambda \sigma / \kappa}{ }^{\alpha}=-1 / 2 \operatorname{curl}\left(\varepsilon^{\delta \gamma \kappa \lambda} C_{\lambda \sigma / \kappa}\right)$. So we can, at least in theory, apply the Green Stokes theorem reducing the problem to a line integral from the bare Eq.(1) through Eq.(4), but we ignore the space dependence of $K_{\mu \nu}$.

The previous Eq.(23a) consists of 16 equations. The $C$ Riemann tensor has been obtained by contraction through

9 We take occasion to remark the evident error of [8] in its notation, in Eq. (4.20b) there the tensor $R_{\alpha \alpha}$ stands for $\Re_{\alpha \alpha}$ the tensor $\epsilon^{\alpha \beta}$ whose skew-symmetry is preserved by a transformation of coordinates $U^{\mu \nu}=\partial x^{\prime \mu} / \partial x^{v}$ such that $U \epsilon U^{+}=\epsilon^{\prime}$. Hence we may say that $K_{\mu \sigma}$ is known to within an anti-symmetric matrix. But this does mean calibrate the constant $S$. In fact, I could choose whatever new $\epsilon$ such that $\epsilon^{\prime}=\lambda \epsilon$ into Eq. (24) for which $S$ could be dimensioned as wanted in the fitting procedure. Nevertheless, once $S$ has been determined in association with a given energy-momentum $T_{\mu \nu}$, I can no longer arbitrarily use any $\epsilon$, but only the one which is bound to that of $S$ by a similarity relation.

This might amaze, that is, the fact that a given cinematic quantity like the energy momentum has been associated with a determined constant tensor like $\epsilon$, but as in geometry I need to adopt a coordinate system and a standard unit to represent a vector, so here to describe his way of being represented in terms of the contraction tensor according the starting position of Eq.(24). On the other hand, we know in physics the case of NeumannFaraday law in which the variation of magnetic flux is related to the electromotive force through the constant 1 in S.I. units. If we had chosen a different factor this would have meant to rescale the electromagnetic constants and the units, so in short $\epsilon^{\alpha \beta}$ gauges $S$. Thus $\epsilon^{\prime}$ is fixed by means of six numbers. Moreover, the 4 conditions of freedivergence of the energy momentum tensor reduce the number of independent equations of Eq.(23a) to 6. Thus the 10 unknown quantities $K_{\mu \nu}$ are defined through 10-6 = 4 accessory conditions that we have chosen as gaussian conditions for the metric tensor.

Since the keplerian motion occurs in a plane and the azimuthal angle is function of the time, and the radial coordinate of the azimuthal angle, the quadruple integral in spherical co-ordinates will be taken on one independent angular variable of the reduced mass. The energymomentum tensor of the single star is $T^{\mu v}(1,2)=d(1,2) u^{\mu} u^{v}, \quad$ with $u^{\mu}$ the 4-velocity components of the $(1,2)$ star mass and $d(1,2)$ density mass which in the case of a punctual mass will be a three dimensional Dirac delta function. Given therefore the keplerian system defined by the equations in polar coordinates $(\rho(1,2), \varphi, \theta)$ :

$$
\rho(1,2)=[m 2 /(m 1+m 2)] \rho
$$

with $\rho=\beta /(1+e \cos \varphi), \quad \beta=a\left(1-e^{2}\right), \quad a$ major semiaxis of the ellipse, $e$ the eccentricity $10, \varphi$ the delayed phase and

$$
\frac{\partial \varphi}{\partial \tau}=\frac{\alpha}{\rho^{2}}, \quad \alpha=\left[G(m 1+m 2) a\left(1-e^{2}\right)\right]^{1 / 2}
$$

the velocity will be taken in the non-relativistic limit so that evaluating $u^{\rho}(1,2)=\partial \rho(1,2) / \partial \tau$ and $u^{\varphi}(1,2)=\partial \varphi / \partial \tau$ the energy-momentum tensor of the system with regard to the centre of mass comes out, taking into account that it equals the sum of the components $T^{\mu v}(1,2)=\mathrm{d}(1,2) u^{\mu}(1,2) u^{v}(1,2)$ :

10 We recall some data of the PSR1913+16 binary system: m1: 1.44 $\mathrm{M}_{\text {sun }}$; $\mathrm{m} 2: 1.38 \mathrm{M}_{\text {sun }} ; e$ : 0.617; orbital period T: 7.72 hours; major semiaxis $a: 1.950 \mathrm{E} 9 \mathrm{~m}$; distance: $5 \div 8 \mathrm{kpc}$; $d \mathrm{~T} / \mathrm{dt}:-2.4056 \mathrm{E}-12$. The axis length comes from the 3th Kepler's law. 


$$
d(1,2)\left[\begin{array}{cccc}
c^{2} & c \frac{m(2,1)}{(m 1+m 2)} \frac{\alpha}{\beta} e \sin \varphi & 0 & c \frac{\alpha}{\rho^{2}} \\
c \frac{m(2,1)}{(m 1+m 2)} \frac{\alpha}{\beta} e \sin \varphi & \frac{m^{2}(2,1)}{(m 1+m 2)^{2}}\left(\frac{\alpha}{\beta} e \sin \varphi\right)^{2} & 0 & \frac{m(2,1)}{(m 1+m 2)} \frac{\alpha^{2}}{\beta \rho^{2}} e \sin \varphi \\
0 & 0 & 0 & 0 \\
c \frac{\alpha}{\rho^{2}} & \frac{m(2,1)}{(m 1+m 2)} \frac{\alpha^{2}}{\beta \rho^{2}} e \sin \varphi & 0 & \frac{\alpha^{2}}{\rho^{4}}
\end{array}\right]
$$

$d(1,2)$ being the point mass density of mass. Moreover, by an integration with respect to the proper time yielding a 4Dirac delta,

it reads as:

$$
T^{\mu v}=\int d \tau\left[\begin{array}{l}
m 1 \delta^{4}\left(\xi-\xi_{1}\right) u^{\mu}(1) u^{v}(1) \\
+m 2 \delta^{4}\left(\xi-\xi_{1}\right) u^{\mu}(2) u^{v}(2)
\end{array}\right] .
$$

By using the method of successive approximations to work out Eq. (23a), we choose $K_{\mu \sigma}=0 \quad$ ( $g_{\mu \sigma}=$ const $=\eta_{\mu \sigma}$ Minkowski cartesian metric $\eta_{00}=-\eta_{11}=-\eta_{22}=$ - $\eta_{33}=1$ ) as zero order approximation. So the metric tensor whereby the indexes are lowered/raised and the matrix of transformation of tensors into spherical coordinates $x^{\prime \rho} \equiv(c t, r, \Theta, \Phi)$ are found as known:

$$
g_{\mu \nu}^{\prime}=\left[\begin{array}{cccc}
1 & 0 & 0 & 0 \\
0 & -1 & 0 & 0 \\
0 & 0 & -r^{2} & 0 \\
0 & 0 & 0 & -r^{2} \sin ^{2} \Theta
\end{array}\right]
$$

$$
\frac{\partial x^{\prime \mu}}{\partial x^{v}}=\left[\begin{array}{cccc}
1 & 0 & 0 & 0 \\
0 & \sin \Theta \cos \Phi & \sin \Theta \sin \Phi & \cos \Theta \\
0 & \frac{\cos \Theta \cos \Phi}{r} & \frac{\cos \Theta \sin \Phi}{r} & -\frac{\sin \Theta}{r} \\
0 & -\frac{\sin \Phi}{r \sin \Theta} & \frac{\cos \Phi}{r \sin \Theta} & 0
\end{array}\right]
$$

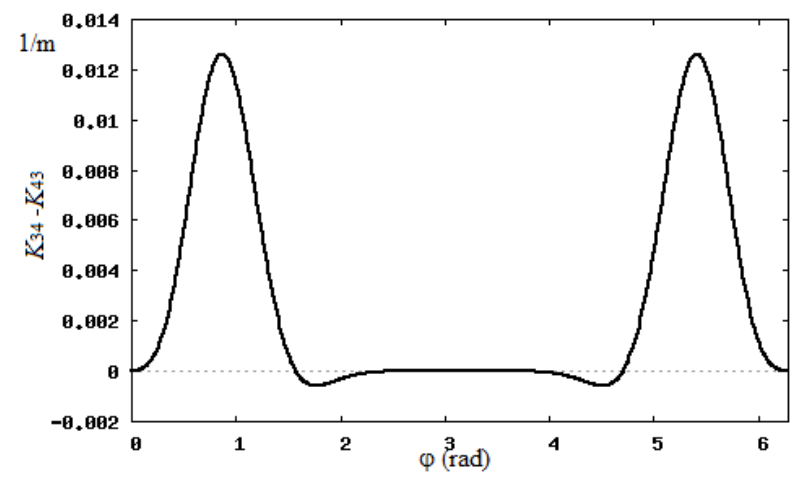

Figure 2a. Symmetry of deformation velocity tensor. Plot in function of the azimuthal angle along the elliptic orbit of the approximation precision $K_{34}-K_{43}$

Moreover, taking into account that $d \tau=(\partial \tau / \partial \varphi) d \varphi$, the proper time in Eq.(26a)-(26b) is parametrized by the azimuthal variable . According to Eq.(24) the zeroth order $C$ Riemann tensor $C_{\mu v}$ will, on using the previous contraction tensor, be of a form something different from an anti-symmetric tensor but this is the rudest approximation. So the skew-symmetry of the $C$ Riemann as well as the symmetry of $K_{\mu v}$ will be considered a goodness index for the nth-order of approximation. As shown below in Figure 2, while the check on the symmetry of the deformation speed of metric, worked out from the integral of Eq.(23a) at orbital distances of PSR1913+16, would seem quite adequate, instead that one on the angular component of $C$ Riemann tensor does not, although behaving well at sidereal distances. However better can be expected at further orders.

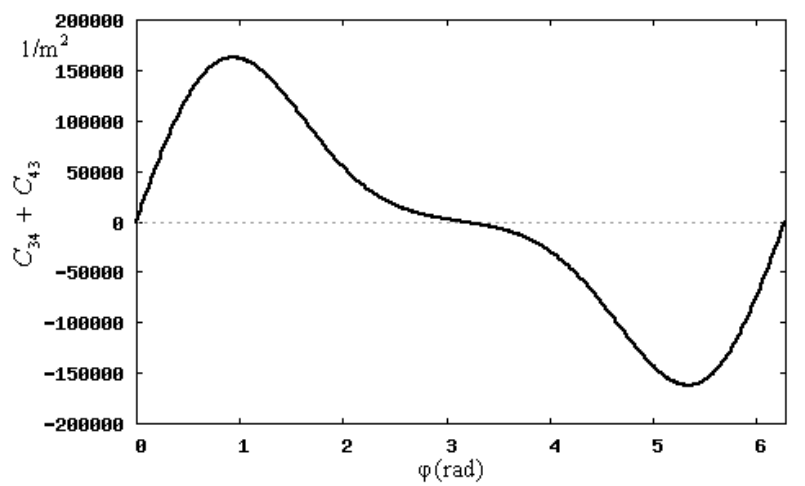

Figure 2b. Skew Symmetry of C Riemann tensor. Plot in function of the azimuthal angle along the elliptic orbit of the approximation precision $\mathrm{C}_{34}+\mathrm{C}_{43}$

To first order we have $K_{\mu \sigma}=y_{\mu \sigma}$. The next step will be, if possible, to determine the new metric

$$
g_{\mu v}\left(x^{\rho} / \tau\right) d \tau=2 c \int K_{\mu v}\left(x^{\rho} / \tau\right) d \tau+\eta_{\mu v}
$$

on the grounds of the definition of the velocity of deformation, so the new trace $r$ and on account of Eq. (24), (25) the new Riemann tensors; hence one determines the kernel simplified of the second term of Eq.(23b) $N_{\sigma \alpha \mu}^{\beta}$, so that the next approximation will be:

$$
K_{\mu \sigma}=\int N_{\sigma \alpha \mu}^{\beta} y_{\beta}^{\alpha} d^{4} \xi+y_{\mu \sigma}
$$

and so on. This operation however has been the biggest difficulty for the presence of the Riemann tensor $R_{\sigma \alpha}{ }_{\alpha \mu}$ in the nucleus of the integral equation. Thus we have tried a further ansatz. Starting as ${ }^{(1)} R_{\mu \nu \rho \sigma}=\left(\epsilon_{\mu \nu} / 4\right) C_{\rho \sigma}$ as alternative first order realization for it, once evaluated the new $g_{\mu \nu}$ by integration of $K_{\mu \sigma}=y_{\mu \sigma} \propto k / S$ according to Eq.(27a), we may assume that the new Riemann tensor will not be dependent to leading order on the factor $k$ nor on $S$ because of the Christoffel symbols that it is composed of, which involves terms of the form ( $\mathrm{g}^{\sigma \mu} \partial_{\alpha}$ $\mathrm{g}_{\beta \sigma}-\ldots$ ) to be derived in turn outputting a term $\alpha / \rho^{2}$ (see Eq.26b1). Hence we have assumed the Riemann tensor be $\propto$ to the $2^{\text {nd }}$ derivative of $C_{\rho \sigma}$ with respect to the time viz. the orbital phase (the only variable that the energymomentum and in Cartesian coordinates the $C_{\rho \sigma}$ depend 
on) owing to Eq.(23c), and therefore it will look like in quasi-cartesian coordinates

as

${ }^{(2)} R_{\mu v \rho \sigma} \approx\left(\alpha / \rho^{2}\right)\left(\epsilon_{\mu \nu} / 4 c^{2}\right) \nabla_{\varphi}{ }^{2} C_{\rho \sigma} / k$, the division by $k$ being due to its structure, see Eq.(2). This way of proceeding may appear very coarse and it is indeed, but it is the most reasonable choice in front of the required memory of some Terabytes for the sheer Riemann tensor.

This done, inserting successively the final worked out tensor metric into the geodesic equation $\partial_{\tau}{ }^{2} x^{\rho}+\Gamma^{\rho}{ }_{\mu \nu} \partial_{\tau} x^{\mu} \partial_{\tau}$ $x^{v}=f^{\rho}$ with $f^{\rho}$ representing a field matter coupling term, would give us the modified acceleration law $\partial_{\tau}{ }^{2} \rho=a(\rho)$ which should fit in as experimental verification with local and solar observations (perihelion precession of planetary orbits, bending of light, though Vortex theory yet include TGR results) as well as galaxies rotation curves. Also Eq. (27b) will be the obliged passage in inferring the cosmological behaviour of fundamental parameters of the evolution of the universe relatively to Friedmann solutions. The treatment of this matter will be object of next publications.

The most great difficulty in expressing the energy loss against the major semi-axis of the elliptic orbit has lain in working out the definite integrals for which numerical methods soon overflow in allocated memory as of wxMaxima software preferred to Maple at this stage. (growing up to nearly $400 \mathrm{pp}$. for single integrand, see the sample in supplementary material : DIFFERENTIAL_ENERGY_LOSS_1_approx.txt -there "f " stands for orbital phase and "th" for $\Theta$ co-latitude). The two software have shown different rounding performances. Adopting numerical algorithms like Cavalieri-Simpson has been therefore necessary (we have taken 30 steps for the integration in $[0,2 \pi]$ with respect to the orbital phase). Nonetheless it has allowed to give an estimate however rough may be of the constant $S$ from known orbital data of PSR 1913+16 [10]. Actually, by differentiating with respect to the time the third Keplero law one gets (mean values for orbit are concerned):

$$
2 T \frac{d T}{d t}=\frac{4 \pi^{2}}{G(m 1+m 2)} 3 a^{2} \frac{d a}{d t} .
$$

On the other hand, differentiating the mean gravitational system energy $<E>=-G m 1 m 2 /(2 r)$ :

$$
\frac{d r}{d t}=\frac{d E}{d t} \frac{2 r^{2}}{G m 1 m 2}
$$

On substituting it into the previous one and taking into account from the free divergence of energy momentum tensor 11 that $-d E / d \tau=c \int t_{s}^{0} n^{s} r^{2} d \Omega$, where for our purpose the energy-momentum tensor is 12 $t_{s}{ }^{0}=\Phi_{s}{ }^{\sigma \alpha} \Phi^{0}{ }_{\sigma \alpha}$, we have obtained $S=1.598 \mathrm{E} 14 \mathrm{~m}^{-1}$ or $S^{-1}=6.26 \mathrm{E}-15 \mathrm{~m}$.

While the mean energy loss of Einstein's theory at $a=$ $1.950 \mathrm{E} 9 \mathrm{~m}$ amounts to $7.959 \mathrm{E} 24 \mathrm{~J} / \mathrm{s}$ - we remember that Einstein averaged emitted power is [11]:

11 We make note in [8] the omission of a $c$ factor in $d E / d \tau$ and a $1 / 4$ in front of the energy momentum.

12 The indexes in the strength tensor are raised by the flat metric rather than the new one (27a) for avoiding overflow error in the calculus. Also, the strength tensor is calculated at the large distance of observation so it looks licit to take an euclidean metric

$$
<P>=\frac{32}{5} \frac{G^{4}}{c^{5}} \frac{m 1^{2} m 2^{2}(m 1+m 2)}{a^{5}\left(1-e^{2}\right)^{7 / 2}}\left(1+\frac{73}{24} e^{2}+\frac{37}{96} e^{4}\right)
$$

-in the Vortex model it equals 1.2715E25 J/s to the first approximation as follows from the expression worked out:

$$
\begin{aligned}
& <P>=\frac{1}{S^{2}}\left(3.642 \cdot 10^{-112}+1.172 \cdot 10^{-114} \sqrt{a}\right. \\
& -\frac{1.077 \cdot 10^{-24}}{\sqrt{a}}+\frac{3.277 \cdot 10^{-21}}{a}+\frac{6.390 \cdot 10^{-4}}{a^{3 / 2}} \\
& +\frac{15.269}{a^{2}}+\frac{2.564 \cdot 10^{31}}{a^{5 / 2}}+\frac{5.858 \cdot 10^{34}}{a^{3}}+\frac{2.430 \cdot 10^{37}}{a^{7 / 2}} \\
& -\frac{3.160 \cdot 10^{56}}{a^{4}}-\frac{5.412 \cdot 10^{60}}{a^{9 / 2}}-\frac{4.745 \cdot 10^{62}}{a^{5}} \\
& -\frac{9.516 \cdot 10^{90}}{a^{11 / 2}}-\frac{9.897 \cdot 10^{93}}{a^{6}}+\frac{4.763 \cdot 10^{95}}{a^{13 / 2}} \\
& +\frac{3.355 \cdot 10^{118}}{a^{7}}+\frac{5.622 \cdot 10^{121}}{a^{15 / 2}}-\frac{3.273 \cdot 10^{124}}{a^{8}} \\
& -\frac{8.715 \cdot 10^{126}}{a^{17 / 2}}-\frac{4.231 \cdot 10^{128}}{a^{9}}-\frac{1.266 \cdot 10^{129}}{a^{19 / 2}} \\
& +\frac{1.282 \cdot 10^{129}}{a^{10}}+\frac{9.631 \cdot 10^{110}}{a^{21 / 2}}+\frac{1.007 \cdot 10^{113}}{a^{11}} \\
& +\frac{2.939 \cdot 10^{108}}{a^{23 / 2}}+\frac{5.856 \cdot 10^{110}}{a^{12}}+\frac{6.325 \cdot 10^{111}}{a^{25 / 2}} \\
& a^{13}
\end{aligned}
$$

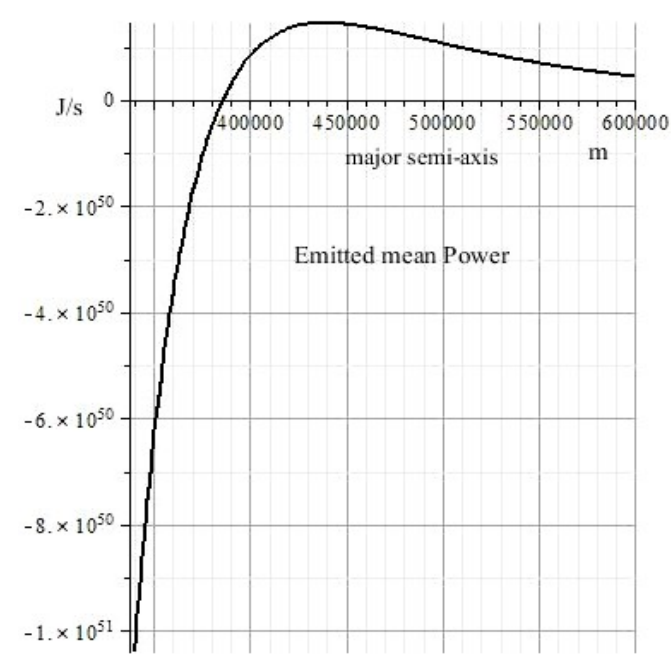

Figure 3. Mean energy loss for Vortex model according to Eq.(29) at reduced distances, much higher than Einstein's amounting to 2E46 J/s. We may note a zero for the value of axis=3.85E5 $\mathrm{m}$ due to the approximation employed, far however from the actual work point interested in

\section{Discussion}

Unfortunately, the previous expression is not monotonous as $1 / a^{5}$ as in Einstein's behaviour and under $4 \mathrm{E} 5 \mathrm{~m}$ shows up a sign variation preventing us from 
yielding a reliable prevision of the constant $S$ like this newfound one at reduced distances. We think of it as due to the early stage of approximation algorithm but successive approximations require very powerful calculus skill. We have also shown in Figure $4 \mathrm{a} / \mathrm{b}$ the differential instantaneous energy loss $d^{2} E / d t d \Omega$ versus the orbital angle in both Vortex and Einstein cases - as well-known the latter proportional to the third derivative of quadrupole moment $Q^{r s}[9]$ :

$$
\begin{aligned}
& \left.W\right|_{\Omega} ^{\text {Einatein }}=\frac{1}{9}\left(\frac{G}{4 \pi c^{5}}\right)\left(\frac{1}{2} \frac{\partial^{3} Q^{r s}}{\partial \tau^{3}} \frac{\partial^{3} Q^{r s}}{\partial \tau^{3}}\right. \\
& -\frac{\partial^{3} Q^{l s}}{\partial \tau^{3}} \frac{\partial^{3} Q^{l m}}{\partial \tau^{3}} n^{s} n^{m} \\
& \left.+\frac{1}{4} \frac{\partial^{3} Q^{l r}}{\partial \tau^{3}} \frac{\partial^{3} Q^{s m}}{\partial \tau^{3}} n^{l} n^{r} n^{s} n^{m}\right) \sin \Theta d \Phi d \Theta \\
& n^{s}=x^{s} / r \\
& Q^{r s}=\int\left(3 x^{\prime r} x^{\prime r}-r^{\prime 2} \delta_{r}^{s}\right) D\left(x^{\prime}\right) d^{3} x^{\prime}
\end{aligned}
$$

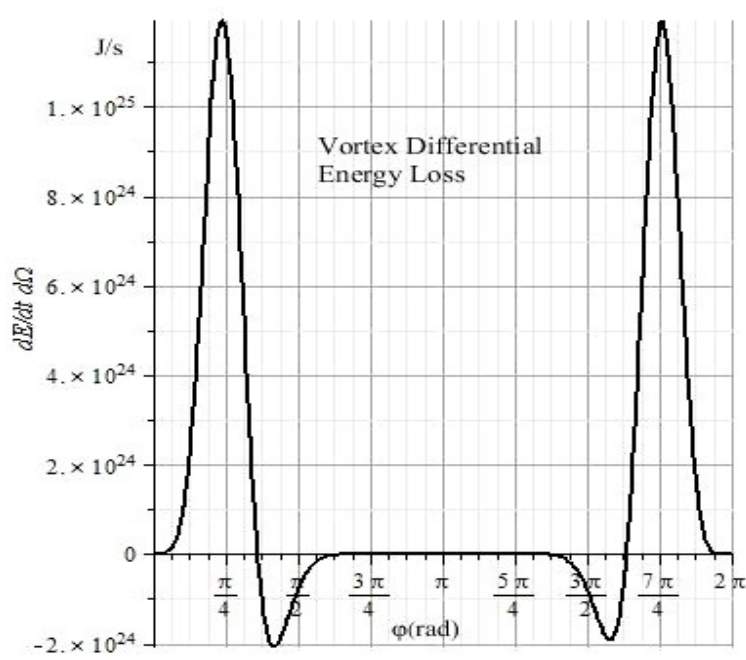

Figure 4(a). Vortex's differential energy loss to $1^{\text {st }}$ approximation. $2 \pi$ periodic instantaneous emitted power vs orbital phase at given direction for polar angle $\Theta=\pi / 2$ and azimuth $\Phi=\pi / 2$. We may note the close resemblance with the Figure 4(b)

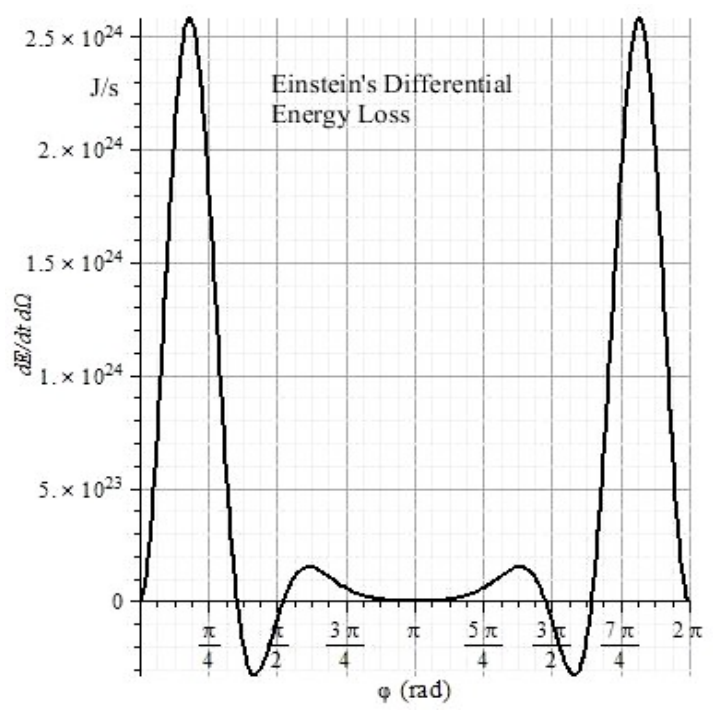

Figure 4(b). Einstein's differential energy loss. $2 \pi$ periodic instantaneous emitted power vs orbital phase at given direction for polar angle $\Theta=\pi / 2$ and azimuth $\Phi=\pi / 2$
As routine, on inserting the mean energy as from the virial theorem into the 3th Kepler's law to cast out the semi-axis $a$ and differentiating, the variation of the orbital period is found as $\dot{T}=\frac{3}{2} \frac{\langle\dot{E}\rangle}{\langle E\rangle} T$, the value of mean energy $E$ averaged on one time period meant as $-\frac{G \cdot m_{1} \cdot m_{2}}{2 \cdot a}$ (however, for ease, just phase average has been taken here dividing the latter by $\left(1-e^{2}\right)$ ). By replacing the values, one gets $\dot{T}=2.40242(2) \mathrm{E}-12$ for TGR versus 2.4096E-12 for the Vortex Model against $\dot{T}^{\operatorname{Exp}}=2.4056(51) \mathrm{E}-12$ (Intrinsic value). [10]. Thus there is an agreement of $0.2 \%$ for the Vortex Model not far from $(0.13 \pm 0.21) \%$ for TGR [10], although for Vortex the fitting procedure must be validated with other binary systems.

We have also reported the orbital period decay advancing at periastron time. Expressing it 13 [15] as $t_{n}-n T=\frac{\dot{T}}{2 T} t_{n}^{2}$ at the modified julian epoch $t_{n}$ (MJD), we are able to collate directly the Vortex Model with data in the matching of the experimental fitted curve, As shown in Figure 5 this latter cannot be distinguished from the Vortex Model one at first sight.
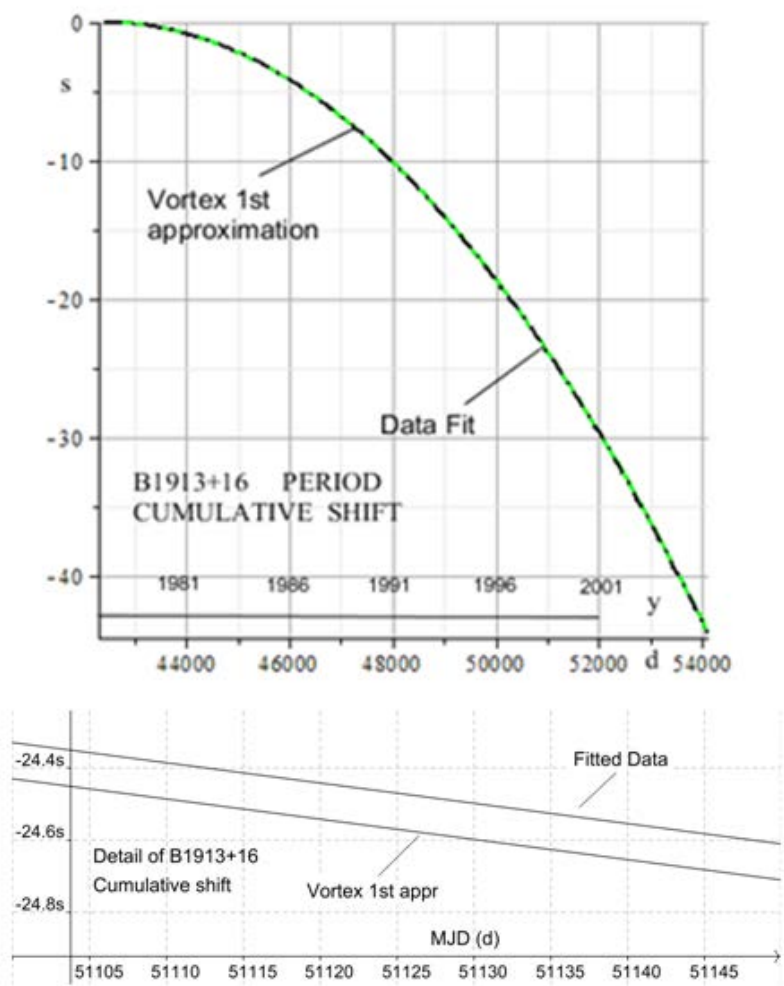

Figure 5. Period change in the damping of B1913+16 system with respect to the fitted data versus Modified Julian Day Time from 1975 to 2005. We can observe how the 1 st approximation Vortex Model approaches so close to the expected decay that cannot be distinguished from it in the upper plot alike the TGR prediction that is dropped out for its challenging matching

13 The formula is got by expressing the accumulated phase as function of time $\Phi(T)=2 \pi \int_{0}^{T} v(t) d t$, expanding the frequency $v$ to 2nd order and drawing out the difference between the times of passage at periastron and the n-th proper period time $n / v_{0}$ 
This result however does not fulfil completely our goals among which there is that of attribute a mass to the graviton through the quantity $h / c S^{-1}$. As anticipation, an attempt to face a $2^{\text {nd }}$ approximation through a more coarse algorithm by dropping the derivatives of ${ }^{(2)} R_{\mu v \rho \sigma}$ $\approx\left(\epsilon_{\mu v} / 4\right) C_{\rho \sigma} / \mathrm{k}$ in the nucleus of Eq.(27b), has furnished the $\langle\dot{E}\rangle=-1.387 \mathrm{E} 25 \mathrm{~J} / \mathrm{s}$ with $S=3.2 \mathrm{E}-1 \mathrm{~m}^{-1}$ with agreement within $5 \%$ to the period decay $\left(\dot{T}_{\text {Vortex }}^{2 n d}=\right.$ 2.628E-12) allowing the graviton to be given the inertial mass of $\sim 1 \mathrm{E}-43 \mathrm{Kg}$.

Finally, in order to detect a $\oplus$ polarized wave whose effect is in-phase deformations of linear distances in a plan normal to the direction of propagation, Michelson interferometer LIGO alike is not suitable in that it works counter-phase. A possible advisable apparatus could be instead a large scale version of the interferometer set up by T.J.Herzog et al. $[13,14]$ in the quantum entanglement equipment making use of a birefringent crystal (e.g. Li $\left.\mathrm{B}_{3} \mathrm{O}_{5}\right)$ the intensity thereof at the output is proportional to $(1+\cos (\Delta \varphi))$ where $\Delta \varphi$ is the overall arms phasedisplacement (Figure 6). Accordingly, if Mirrors 1 and 2 moved in opposite direction as expected in TGR wave detectors, the output would be the same, because the phases are summed up in the $\Delta \phi$ term. Not so evidently in Vortex theory since they are expected to stretch or shrink in phase along the arms.

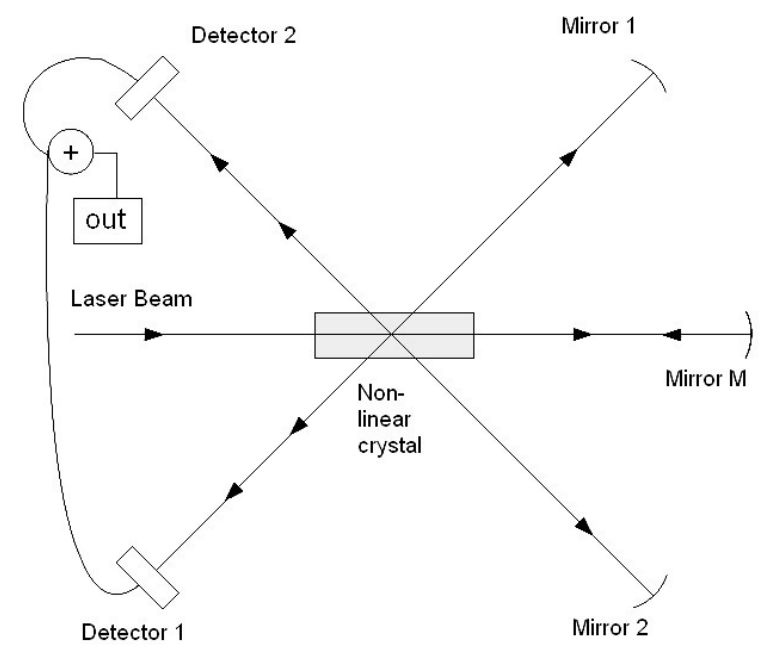

Figure 6. Scheme of interferometric antenna for detection of gravitational wave in the $\oplus$ polarization in which a linear crystal splits the incident beam in two ones forth and back

As a simple order of magnitude of the expected effects we need to evaluate the integral of Eq. (14) meant as deviation from the pseudo-euclidean metric to the distance from earth. This has yielded a value about $1 \mathrm{E}-21$ for the $\oplus$ polarization somewhat the same than TGR, because of the quantum limits of the interferometer for which we can use the computation made for the Michelson- Morley's one in [[9], sec.5.7], which predicts a minimum amplitude detectable of $\Delta=\sqrt{\frac{\hbar \tau}{m L^{2}}}, L$ standing for arm length, $m$ for the mirror mass and $\tau$ for the measure elapsing time.

\section{Conclusions}

We have described a gravitational propagation framework that we shall expect to be appropriate in the theoretical interpretation of gravitational wave detection data, in which perturbations of metric produced by a source are detected over macroscopic scale like interferometry. We have shown that taking $K_{\mu v}$ as a symmetric potential of a massless 2 spin particle (graviton), has recovered the two independent polarizations of the field as in Einstein's theory, but with the difference that counter-phase effects are checkable only in the $\otimes$ polarization. Great difficulty has been encountered in resolving the field equations, which has made very hard the verification of the B1913+16 experimental data through ordinary numerical algorithms, so limiting the analysis to the leading approximation. Certainly, higher order approximations are mandatory to make a telltale check of the theory; nevertheless for our purposes it is sufficient to be acquainted with the possibility of the evaluation of the coupling constant $S=1.6 \mathrm{E} 14 \mathrm{~m}^{-1}$ as a measure of the intrinsic inertia of the Space-Time to sweep out gravitational radiation and to assure causality in relativity as stressed in [8]. Comparable agreement has been shown between the Vortex Model and the Einstein energy losses amount and even an excellent fit to B1913+16 period decay to within $0.2 \%$. However, this value is affected by the uncertainty of the coarse approximation in working out the integral equation for computing $K_{\mu \nu}$ in Eq.(27b) and by that of the large distance of the system obtained by dispersion measures, although in the exact theory the integrated emitted power should not depend on it, and so has proved this approach. As anticipation a $2^{\text {nd }}$ order coarse solution performed entirely by Maple 15, anyway, has come by a value of $S=0.32 \mathrm{~m}^{-1}$ fitting the period decay to within 5\%. Accordingly this evaluation must be retained only for defining a magnitude order to play with. Therefore, further confirmations especially to the $2^{\text {nd }}$ order are expected from paralleling other binary systems as the brighter B1534+12 and the promising relativistic one NSNS BJ0737.

\section{Acknowledgment}

I am grateful to wxMaxima developers for their great software and to CILEA (now CINECA) supercomputer section.

\section{References}

[1] Ferrarese, G.: Lezioni di Relatività Generale, chap.1,, §5 and §7. Pitagora Editrice, Bologna, 2001; also for details.: Ferrarese, G.., Stazi, L.: Lezioni di Meccanica Razionale vol.1 Ch.1,4.7 Pitagora Ed., Bologna, 1989.

[2] Cattaneo, C., Introduction à la Théorie Macroscopique des Fluides Relativistes, Lectures held at the Collège de France, italian translation, Pitagora Ed. Bologna, 1980.

[3] Ferrarese, G., "Compatibility Conditions in Relativistic Continuum Dynamics", in "Advances in modern continuum dynamics, Proc. of the Int. Conf. At Elba Island"', G.Ferrarese Ed., Bologna, Pitagora, 1992, pp.161-172.

[4] Ferrarese, G., Stazi, L., Lezioni di Meccanica Razionale vol 2 chap.VIII, §1.9-10, Pitagora Ed., Bologna, 1989, pp. 591-598.(cf. also Ferrarese,G.: Lezioni di Meccanica superiore, Veschi. Roma, 1968-off printing.)

[5] Ferrarese, G., Stazi, L., "Il teorema di Lagrange nei fluidi perfetti relativistici”, Rendiconti di Matematica 3, 1989, pp. 409-421. 
[6] Ferrarese, G., Stazi, L., Lezioni di Meccanica Razionale vol 2 chap.VIII, §3.2, Pitagora Ed., Bologna, 1989, pp. 616-619.

[7] Sokolnikoff, I.S., Tensor Analysis, Chap. 6, §105, Wiley, New York, 1951.

[8] Tailherer, M., "A Critical reading on the theory of gravitational wave propagation”, Journal of Physical \& Natural Sciences, Vol.1, Issue $\quad 1, \quad 2007 \quad$ [Online]. www.scientificjournals.org/journals2007/articles/1085.pdf.

[9] Ohanian, H.C., Ruffini, R., Gravitation and Spacetime, Chap. 6.6; 5.2. W.W. Norton \& Company, 1994.

[10] Weisberg J. M., Taylor, J. H., "Relativistic binary pulsar B1913+16: thirty years of observations and analysis", arXiv: astro-ph/0407149 v1, 2004. [Online].
[11] Peters, P.C., Mathews, J., “Gravitational radiation for point masses in a keplerian orbit”, Physical Review, 131, 1963, 435-440.

[12] Herzog,T.H.,Kwiat,P.G., et al., Phys. Rev. Letters, 75: 3034, 1995.

[13] Gasiorowicz,S., Quantum Physics, 3th edition, Ch. 20.2 , Wiley, N.Y., 2003.

[14] Loinger, A., "GW's towards fundamental principles of GR", arXiv:physics/0709.0490 v1,2007, and references therein. See also arXiv:astro-ph/9904207,1999. [Online].

[15] Maggiore, M., Gravitational Waves, vol. 1, §6.2.3, OUP Oxford, 2007, pp. 313.322-326.

[16] Kar, S., Sengupta, S.: The Raychaudhuri equations: A brief review, arXiv:gr-qc/061123 v1, 2006. [Online]. 